\title{
Stable Length Estimates of Tube-like Shapes *
}

\author{
Herbert Edelsbrunner $^{\dagger}$ and Florian Pausinger ${ }^{\dagger}$
}

\begin{abstract}
Motivated by applications in biology, we present an algorithm for estimating the length of tube-like shapes in 3dimensional Euclidean space. In a first step, we combine the tube formula of Weyl with integral geometric methods to obtain an integral representation of the length, which we approximate using a variant of the Koksma-Hlawka Theorem. In a second step, we use tools from computational topology to decrease the dependence on small perturbations of the shape. We present computational experiments that shed light on the stability and the convergence rate of our algorithm.
\end{abstract}

Keywords. Differential geometry, integral geometry, quasi-Monte Carlo integration, tubes, Quermassintegrals, discrepancy, persistent homology, algorithms, stability, convergence.

\section{Introduction}

The length of a curve in Euclidean space is an elementary geometric concept, and it is well defined provided the curve is not wild. We consider the problem of computing the length of curve- or tube-like shapes, such as root systems of plants. Branching is allowed, but the real difficulty lies in the small but positive thickness, which renders length an undefined concept, at least in the mathematical sense. One may want to construct a 1-dimensional skeleton and then take the length, but this construction is instable; see [4, 9]. Instead of stabilizing the skeleton, we aim at estimating the length of a hypothetical skeleton, which we leave unspecified. The difficulty in the related case of a coastline, studied famously by Mandelbrot [19], is the dependence on the resolution to which the curve is being measured. The length diverges as the resolution increases, suggesting the dimension of the coastline be larger than 1.

${ }^{*}$ This research is partially supported by the National Science Foundation (NSF) under grant DBI-0820624, the European Science Foundation (ESF) under the Research Network Programme, the European Research Council (ERC) under the Toposys project FP7-ICT-318493-STREP, and the Russian Government under the Mega Project 11.G34.31.0053.

${ }^{\dagger}$ IST Austria (Institute of Science and Technology Austria), Klosterneuburg, Austria.
Noticing the abundance of tube-like shapes in nature and therefore in the sciences, we aim at producing a length estimate that is stable under perturbations of the shape. We believe that this will be useful in the study of geographic structures, including river and road networks, as well as biological and medical structures, including trees, blood vessels, nerve cells, and more. Our length estimation algorithm combines intuitive geometric ideas with topological methods:

1. using the formula of Weyl [13, 24, 26], it expresses the length of a tube-like shape by an integral geometric representation of the second Quermassintegral;

2. applying a recent version of the Koksma-Hlawka Theorem $[14,15,17]$, it approximates the resulting integral with explicit bounds on the integration error;

3. exploiting insights into the persistence diagram of a height function $[5,6,12]$, it gives a length estimate that is stable under perturbations.

We implement the algorithm and analyze its performance. Our experiments give clear evidence for the effectiveness of the topological method and the stability of the length estimate provided by our algorithm.

Outline. In Section 2, we introduce the tube formula of Weyl and integral geometric tools to represent and study tube-like shapes. In Section 3, we express the length of tubelike shapes with quasi-Monte Carlo methods. In Section 4, we improve the stability using methods from computational topology. In Section 5, we turn the expression of length into an algorithm and analyze its performance. Section 6 concludes the paper.

\section{Tubes and Integral Geometry}

In this section, we study and extend special cases of the tube formula of Weyl [13, 20, 26]. This formula holds for general smooth submanifolds of a finite-dimensional Euclidean space. The main result of this section is a simple relationship 
between the geometric properties of a curve and its thickened version in $\mathbb{R}^{3}$ :

$$
\begin{aligned}
& L=Q_{2} / \pi ; \\
& L=\left[Q_{2}+\left(4+C_{0}\right) k r_{0}-2 \pi \ell r_{0}\right] / \pi .
\end{aligned}
$$

Equation (1) holds for a smooth closed curve of length $L$, in which $Q_{2}$ is the total mean curvature of the surface bounding a sufficiently thin uniform thickening of the curve. Equation (2) generalizes (1) to a curve with $k \geq 0$ right-angled forks and $\ell \geq 0$ tips. Indeed, the compensation terms are exact if each fork is a T-junction in which the main branch is locally straight and the side branch grows out locally straight and at a right angle to the main branch. The constant, $C_{0}=3.459 \ldots$, is a definite integral that will be explained in Section 2. Note that (1) is independent of the thickening radius, provided it is sufficiently small so that the surface bounding the tube is smoothly embedded in $\mathbb{R}^{3}$. In contrast, (2) depends on the radius, $r_{0}$. In practice, $r_{0}$ is usually difficult to estimate, but for small values of $k, \ell$, and $r_{0}$, the error caused by setting $r_{0}$ to zero is negligible.

Closed curves. Let $\Gamma$ be a closed and smoothly embedded curve in $\mathbb{R}^{3}$. Given $r+\varepsilon \geq 0$, the uniformly thick tube defined by the curve and the radius is the set $\Gamma_{r+\varepsilon}$ of points $x$ at Euclidean distance at most $r+\varepsilon$ from some point of $\Gamma$. Equivalently, it is the union of closed balls with radius $r+\varepsilon$ whose centers lie on $\Gamma$. For $r+\varepsilon$ small enough such that each point in $\Gamma_{r+\varepsilon}$ has a unique closest point on the curve, the boundary of the tube is a smoothly embedded surface. In this case, the volume of the tube is the length of the curve times the area of a cross-sectional disk:

$$
\begin{aligned}
\operatorname{vol} \Gamma_{r+\varepsilon} & =L \pi(r+\varepsilon)^{2} \\
& =L \pi r^{2}+2 L \pi r \varepsilon+L \pi \varepsilon^{2} .
\end{aligned}
$$

To prepare the next step, let $\mathbb{M}=\Gamma_{r}$. Assuming $r$ and $\varepsilon$ are both sufficiently small, we can use the formula of Weyl to compute the volume of $\mathbb{M}_{\varepsilon}=\Gamma_{r+\varepsilon}$. Specifically, there are constants $Q_{0}, Q_{1}, Q_{2}, Q_{3}$ such that the volume of this uniformly thick tube is

$$
\operatorname{vol} \mathbb{M}_{\varepsilon}=Q_{0}+Q_{1} \varepsilon+Q_{2} \varepsilon^{2}+Q_{3} \varepsilon^{3} .
$$

Since the body thickened by $\varepsilon$ is equal to the curve thickened by $r+\varepsilon$, we may compare (5) with (4) and get

$$
\begin{array}{ll}
Q_{0}=L \pi r^{2}, & Q_{1}=2 L \pi r, \\
Q_{2}=L \pi, & Q_{3}=0 .
\end{array}
$$

We extract Equation (1) for the case of a uniformly thickened closed curve from the third relation. While we could compute the length also from the first two relations, the third relation has the advantage to be independent of the radius, $r$.

Curves with ends. Next, let $\Gamma \subseteq \mathbb{R}^{3}$ be a curve with endpoints. Thickening the curve uniformly, we get a tube with two halves of a ball attached at the ends. Similar to before, we assume that $r+\varepsilon$ is small enough such that the surface of the thickened tube is embedded in $\mathbb{R}^{3}$. The volume is the length times the area of a cross-sectional disk plus the volume of a complete ball:

$$
\operatorname{vol} \Gamma_{r+\varepsilon}=L \pi(r+\varepsilon)^{2}+\frac{4 \pi}{3}(r+\varepsilon)^{3} .
$$

Setting $\mathbb{M}=\Gamma_{r}$, as before, and computing the volume of $\mathbb{M}_{\varepsilon}=\Gamma_{r+\varepsilon}$ with the tube formula of Weyl in (5), we get

$$
\begin{array}{ll}
Q_{0}=L \pi r^{2}+\frac{4 \pi}{3} r^{3}, & Q_{1}=2 L \pi r+4 \pi r^{2}, \\
Q_{2}=L \pi+4 \pi r, & Q_{3}=\frac{4 \pi}{3} .
\end{array}
$$

Using the third relation, we get Equation (2) with $k=0$ and $\ell=2$ for a uniformly thickened curve with two ends.

Curves with forks. Finally, we consider a curve with a fork, restricting ourselves to one in which the side-branch leaves the main fork at a right angle. It is convenient to first study a cross in which two curves intersect each other at a right angle. Assume that both curves are locally straight and that they are thickened to tubes with unit radius. The surface bounding the union of the two tubes has two elliptic creases that delimit the submerged patches on the cylinders. Since these patches do not bound, the surface area of the union is less than the sum of the surface areas of the two tubes. To compute by how much, we take the two cylindrical pieces of length 2 near the intersection, and compute the area as the disjoint union of eight triangular wedges, each with a base that goes half-way around a cylinder and with height 1; see the dark shaded triangles in Figure 1. To do

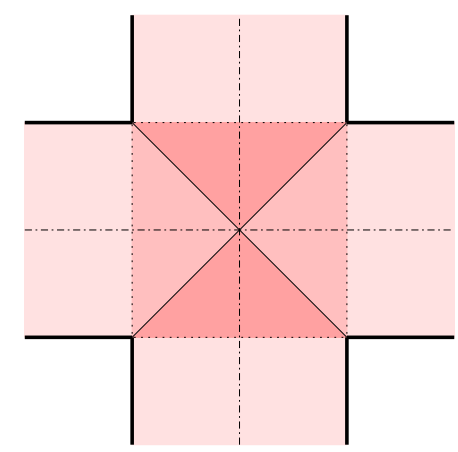

Figure 1: A cross formed by two locally straight curves meeting at a right angle. We see four of the eight wedges that constitute the surface near the intersection.

the computations, we represent the cross-section of a cylinder as the graph of the function $f(x)=\sqrt{1-x^{2}}$, get $f^{\prime}(x)=$ $-x / \sqrt{1-x^{2}}$, and note that $\sqrt{1+f^{\prime}(x)^{2}}=1 / \sqrt{1-x^{2}}$. Substituting $x=\sin \alpha$, we get $\mathrm{d} x=\cos \alpha \mathrm{d} \alpha$ and

$$
\int_{x=-a}^{a} \frac{\mathrm{d} x}{\sqrt{1-x^{2}}}=\int_{\alpha=-\arcsin a}^{\arcsin a} \mathrm{~d} \alpha
$$


which evaluates to $2 \arcsin a$. With these notions, we compute the area of a wedge by integrating the length of the circular cross-section along the axis of the cylinder:

$$
\begin{aligned}
\text { Area }_{W} & =\int_{y=0}^{1} \int_{x=-y}^{y} \sqrt{1+f^{\prime}(x)^{2}} \mathrm{~d} x \mathrm{~d} y \\
& =2 \int_{y=0}^{1} \arcsin y \mathrm{~d} y \\
& =2\left[\sqrt{1-y^{2}}+y \arcsin y\right]_{0}^{1},
\end{aligned}
$$

which evaluates to $\pi-2$. Multiplying with 8 , we get the area of the cross equal to $8 \pi-16$, which is 16 less than the area of the two cylindrical pieces. The loss of area for a fork is half that for a cross, namely 8 . Generalizing to tubes of radius $r$, that loss is $8 r^{2}$. The loss in total mean curvature is calculated in two increments. The first is the (positive) mean curvature integrated over the lost area, which is $8 r^{2} / 2 r=4 r$. The second increment is the (negative) contribution along the elliptic creases, which for unit radius is minus four times the contribution of a quarter ellipse, which is

$$
C_{0}=4 \int_{0}^{\sqrt{2}} \frac{\arccos \left(f(x)^{2}\right)}{2} \sqrt{1+f^{\prime}(x)^{2}} \mathrm{~d} x
$$

where the graph of $f(x)= \pm \sqrt{1-x^{2} / 4}$ is the ellipse, and $\arccos \left(f(x)^{2}\right)$ is the angle between the normal vectors of the two cylinders at the point $(x, f(x))$ on the ellipse. Evaluating this integral, we get $C_{0}=3.459 \ldots$ In summary, for a rightangled fork of radius $r$, we need to add $4 r+C_{0} r$ to get the total mean curvature of the two cylindrical surfaces from which we can then estimate the length of the curve that generates the tube; see Equation (2).

Quermassintegrals. The coefficients in (5) have an integral geometric meaning. Call a compact subset of $\mathbb{R}^{3}$ a body if it is equal to the closure of its interior. For example, the tube, $\mathbb{M}$, obtained by thickening a closed curve by $r>0$ is a body. For $0 \leq i \leq 3, Q_{i}$ is a constant times the $i$-th Quermassintegral of $\mathbb{M}$ in $\mathbb{R}^{3}$ :

$$
Q_{i}=c_{i} \cdot \int \chi(\mathbb{M} \cap P) \mathrm{d} P,
$$

in which the integral is over all $i$-planes $P$ in $\mathbb{R}^{3}$, and $\chi$ denotes the Euler characteristic of the intersection of $\mathbb{M}$ with the $i$-plane $P$. The $c_{i}$ are independent of $\mathbb{M}$, so we can compute them from the Quermassintegrals of the unit ball [20, Chapter 17]. Thickening by $\varepsilon$, we get the volume as a cubic polynomial in $\varepsilon$; see (7), with the coefficients of the monomials given in the first row of Table 1 . Next, we determine the Quermassintegrals, which we get by dividing the coefficients in the first row through the constants in the second row of Table 1 . For $i=0$, the integral is over all points in $\mathbb{R}^{3}$. The measure of the points in the ball is its volume, $\frac{4 \pi}{3}$, which gives $c_{0}=1$. For $i=1$, the integral is over all lines,

\begin{tabular}{c|cccc} 
& $i=0$ & 1 & 2 & 3 \\
\hline$Q_{i}$ & $4 \pi / 3$ & $4 \pi$ & $4 \pi$ & $4 \pi / 3$ \\
$c_{i}$ & 1 & $2 / \pi$ & 1 & $4 \pi / 3$
\end{tabular}

Table 1: The first row lists the coefficients of the monomials in the volume polynomial of the unit ball. The second row lists the constants that relate the coefficients with the Quermassintegrals.

which we parametrize by a direction $u \in \mathbb{S}^{2}$ and a point in the plane normal to the direction. The measure of lines that have a non-empty intersection with the ball is $2 \pi^{2}$, which gives $c_{1}=2 / \pi$. For $i=2$, the integral is over all planes, which we parametrize by $u \in \mathbb{S}^{2}$ and the distance from the origin. The measure of planes with non-empty intersection with the ball is $4 \pi$, which gives $c_{2}=1$. Finally, for $i=3$, we have only one 3-plane, namely $\mathbb{R}^{3}$ itself, so the integral is 1 , which gives $c_{3}=4 \pi / 3$.

Our primary interest is in the case $i=2$, for which $Q_{2}$ is the total mean curvature of the boundary of $\mathbb{M}$. Among the different approaches to computing the total mean curvature of that surface, we prefer the integral geometric approach because it has an alternative topological interpretation that leads to a stable length estimate of tube-like shapes.

\section{Quasi Randomness}

In order to evaluate the second Quermassintegral, we apply a version of the Koksma-Hlawka Theorem recently proved by Harman [14]. This theorem explicitly bounds the integration error,

$$
\operatorname{Err}(N, X)=\left|\frac{1}{N} \sum_{j=1}^{N} F\left(x_{j}\right)-\int F(x) \mathrm{d} x\right|,
$$

in which $X=\left\{x_{1}, x_{2}, \ldots, x_{N}\right\}$ contains the first $N$ points of an infinite sequence. It separates the contributions of the variation of the function and the distribution properties of the points at which the function is evaluated. We therefore introduce the Harman variation of a function as well as concepts from uniform distribution theory before stating the algorithm for estimating the length of tube-like shapes.

Discrepancy. Let $X=\left(x_{j}\right)_{j \geq 1}$ be an infinite sequence of points in the unit interval $[0,1]$, and let $J$ be a closed interval contained in $[0,1]$. For every $N \geq 1$, let $\#(J, N, X)$ denote the number of indices $j \leq N$ for which $x_{j} \in J$. The sequence is uniformly distributed if

$$
\lim _{N \rightarrow \infty} \frac{\#(J, N, X)}{N}=\text { length } J
$$

for every interval $J \subseteq[0,1]$. This definition can be extended to sequences of points, $X$, in the $s$-dimensional unit cube, $[0,1]^{s}$. The discrepancy quantifies the irregularity of a finite set or an infinite sequence of points. Letting $\mathcal{K}$ be the set of all convex subsets of $[0,1]^{s}$, the isotropic discrepancy is

$$
\operatorname{Dis}_{\mathcal{K}}(N, X)=\sup _{K \in \mathcal{K}}\left|\frac{\#(K, N, X)}{N}-\operatorname{vol} K\right| \text {. }
$$


An (axis-parallel) box is the Cartesian product of $s$ intervals, one in each dimension. Restricting the set of convex subsets to boxes, we define the extreme discrepancy, $\operatorname{Dis}_{\mathcal{B}}(N, X)$, in which $\mathcal{B}$ denotes the set of all boxes contained in $[0,1]^{s}$. Clearly, $\operatorname{Dis}_{\mathcal{B}}(N, X) \leq \operatorname{Dis}_{\mathcal{K}}(N, X)$ since $\mathcal{B} \subseteq \mathcal{K}$. Furthermore, we have $\operatorname{Dis}_{\mathcal{K}}(N, X) \leq$ const $\cdot \operatorname{Dis}_{\mathcal{B}}(N, X)^{1 / s}$; see $[18,21]$, in which the exponent $1 / s$ is best possible due to an example of Zaremba [27].

As mentioned, the Koksma-Hlawka Theorem bounds the integration error by a product of two terms, the second being the discrepancy of the point set. This motivates us to look for sequences whose discrepancy is as small as possible. For the purposes of this paper, the isotropic discrepancy is the measure of choice. For $s \geq 2$, the best lower bounds for any point set are due to Schmidt [23], and the best upper bounds for $N$ independent random variables uniformly distributed over the unit cube are due to Stute [25] for $s>2$, and Beck [2] for $s=2$ :

$$
\frac{1}{\text { const } \cdot N^{\frac{2}{s+1}}} \leq \operatorname{Dis}_{\mathcal{K}}(N, X) \leq \frac{\text { const } \cdot N^{\varepsilon}}{N^{\frac{2}{s+1}}}
$$

in which $\varepsilon>0$ and $N>N_{0}(s, \varepsilon)$. As a comparison, we mention that there are deterministic sequences with extreme discrepancy at most some constant times $(\log N)^{s} / N$, and this is conjectured to be best possible. The expected extreme discrepancy of a set chosen uniformly at random is a constant divided by $N^{1 / 2}$, for every constant dimension $s$. For concrete examples of deterministic low discrepancy sequences and their use in practice, we refer to the recent monograph [10]. We also refer to [1], in which the problem of sampling on a sphere is reduced to the study of point sets in the plane with small isotropic discrepancy.

Variation. The original version of the Koksma-Hlawka Theorem is stated in terms of the Vitali variation of a function $F:[0,1]^{s} \rightarrow \mathbb{R}$; see [15]. To compute it, we take the alternating sum of function values at the corners of an axisparallel box, we take the sum over all boxes in a decomposition of $[0,1]^{s}$, and we finally take the supremum over all such decompositions. More precisely, the theorem was proved for the Hardy-Krause variation, which is the sum of Vitali variations over the restrictions of $f$ to all faces of the cube, including $[0,1]^{s}$ itself. The functions that arise in this paper include steps of discontinuity, for which the Vitali variation can be unbounded.

For such functions, the Harman variation is more appropriate. Let $F$ be a bounded measurable function, and write $\min F$ and $\max F$ for the smallest and the largest function value. If the superlevel sets of $F$ at $c$ can be written as an algebraic sum of finitely many convex sets, we denote the minimum number of convex sets required to do so with $\operatorname{har}(F, c)$. More specifically, this means that the indicator function of $F^{-1}[c, \infty)$ is the difference between two sums of indicator functions of convex sets. Note that $\operatorname{har}(F, c)$ is undefined if the corresponding superlevel set can not be written as an algebraic sum of finitely many convex sets. If $\operatorname{har}(F, c)$ is defined for all $c$, then the Harman variation of $F$ is given by

$$
\operatorname{Har}(F)=\int_{c=\min F}^{\max F} \operatorname{har}(F, c) \mathrm{d} c,
$$

provided the right hand side exists as a Riemann integral; see [14].

Parametrization. To write the second Quermassintegral in (14) in terms of a function on a unit cube, we need to parametrize the 2-planes, which we do using the signed distance from the origin, $\varrho$, and the normal direction. Each direction can be specified by a value $a \in[-1,1]$ along a diameter of the unit sphere, and an angle $\varphi \in[0,2 \pi]$ within the plane normal to that diameter. By Archimedes' Theorem, picking $a$ and $\varphi$ uniformly in their respective intervals gives a point uniformly sampled on the sphere. We thus obtain a parametrized version of the third integral in (14):

$$
Q_{2}=\int_{\varphi=0}^{2 \pi} \int_{a=0}^{1} \int_{\varrho=-\infty}^{\infty} \chi(\mathbb{M} \cap P) \mathrm{d} \varrho \mathrm{d} a \mathrm{~d} \varphi
$$

where $P$ is of course the 2-plane specified by $\varrho, a$, and $\varphi$. Note that we integrate only over $a \in[0,1]$, effectively sampling only half the unit sphere. We do this to compensate for the fact that each 2-plane has two normal directions, here represented by antipodal points on the sphere.

In the application below, we write the triple integral as a double integral of the function

$$
F(a, \varphi)=\int_{\varrho=-\infty}^{\infty} \chi(\mathbb{M} \cap P) \mathrm{d} \varrho .
$$

We do this for several reasons, the most important being the link to persistence, as discussed in Section 4. Another is the property discussed next.

Sampling directions. To make the connection to the Quermassintegral for $i=2$, we argue that the Harman variation of the function $F$ is bounded. Here, we assume that $\mathbb{M}$ is the underlying space of a simplicial complex with a finite number of vertices, and its diameter is bounded.

VARiation Lemma. Let $\mathbb{M}$ be the underlying space of a simplicial complex with $m$ vertices in $\mathbb{R}^{3}$, and let $F$ be the function in (21). Then the Harman variation of $F$ is bounded from above by a constant times $m^{6} \operatorname{diam} \mathbb{M}$.

Proof. We do the proof in two steps, first analyzing the function on the sphere, and second mapping the sphere to a rectangle in the plane.

For the first step, let $G(u)$ be the integral of $\chi(\mathbb{M} \cap P)$ over all planes $P$ with normal direction $u$. Furthermore, let $f_{u}(v)=\langle u, v\rangle$ be the height of a point $v$ in the direction $u \in \mathbb{S}^{2}$. We begin by decomposing the directions in the northern hemisphere into regions within which the ordering of the 
vertices by height is constant. This decomposition is obtained by cutting the hemisphere with the $\left(\begin{array}{c}m \\ 2\end{array}\right)$ planes passing through the origin whose normals are differences between pairs of the $m$ vertices of $\mathbb{M}$. This gives an arrangement of $\left(\begin{array}{c}m \\ 2\end{array}\right)$ half-great-circles forming fewer than $m^{4} / 4$ spherical regions, which we refer to as chambers. Within each chamber, the function $G$ has a particularly simple form, as we now explain. Fixing a chamber and a direction $u$ in its interior, we index the vertices such that $f_{u}\left(v_{1}\right)<f_{u}\left(v_{2}\right)<\ldots<f_{u}\left(v_{m}\right)$. Let $P_{i}$ be the plane of points $\langle x, u\rangle=\frac{1}{2} f_{u}\left(v_{i}\right)+\frac{1}{2} f_{u}\left(v_{i+1}\right)$, and define $\chi_{i}=\chi\left(\mathbb{M} \cap P_{i}\right)$. With these notions, we can write the function value at $u$ as

$$
G(u)=\sum_{i=1}^{m-1} \chi_{i} \cdot\left(f_{u}\left(v_{i+1}\right)-f_{u}\left(v_{i}\right)\right) .
$$

While this formula holds for all directions in this chamber, we get different function values because the directional distance between contiguous vertices depends on the direction and not just the chamber. To overcome this difficulty, we interpret $G(u)$ as the directional length of a single vector in $\mathbb{R}^{3}$, namely of

$$
w=\sum_{i=1}^{m-1} \chi_{i} \cdot\left(v_{i+1}-v_{i}\right) .
$$

Observe that $G(u)=\langle u, w\rangle$. Importantly, $w$ depends on the chamber but not on the direction. Letting $\psi$ be the angle between $u$ and $w$, we have $G(u)=\|w\| \cdot \cos \psi$. Given a threshold, $c \in \mathbb{R}$, the superlevel set, $G^{-1}[c, \infty)$, consists of all directions $u$ with angle $\psi \leq \arccos \frac{c}{\|w\|}$. These directions form a spherical cap on $\mathbb{S}^{2}$. In summary, each superlevel set of $G$ is the union of fewer than $m^{4} / 4$ regions with disjoint interiors, each the intersection of spherical caps. Indeed, a single such region is the intersection of a spherical cap with the corresponding chamber, which itself is the intersection of a possibly large number of spherical caps. However, the total number of caps is less than $3 \mathrm{~m}^{2} / 2$, namely two for each arc and one for each chamber in the arrangement.

In the second step, we map the northern hemisphere to $[0,1] \times[0,2 \varphi]$ such that $F(a, \varphi)=G(u)$ whenever $u$ maps to $(a, \varphi)$. This map has interesting properties, which have recently been studied in [1, Lemma 3] to give discrepancy bounds for caps on a sphere. Specifically, they show that the image of a cap is the algebraic sum of a constant number of convex sets in the plane. With this insight, we are now ready to bound the Harman variation of the function $F$. The integral of $\operatorname{har}(F, c)$ over all superlevel sets restricted to one chamber is some constant, times the number of caps needed to describe the restriction, times the length of $w$, where we get the constant from the analysis in [1]. The length of $w$ is bounded from above by the maximum possible absolute $\mathrm{Eu}-$ ler characteristic times the diameter of $\mathbb{M}$. The Euler characteristic cannot be larger in magnitude than $3 \mathrm{~m}^{2} / 2$. To see this, we note that the simplicial complex defining $\mathbb{M}$ has fewer than $m^{2} / 2$ edges. The intersection with a plane has therefore fewer than $m^{2} / 2$ vertices, and because of planarity, it has fewer than $3 \mathrm{~m}^{2} / 2$ edges and fewer than $\mathrm{m}^{2}$ faces. The Euler characteristic is the number of vertices minus the number of edges plus the number of faces, which is a number between minus and plus $3 m^{2} / 2$. Taking the sum over all chambers gives

$$
\operatorname{Har}(F) \leq \text { const } \cdot \frac{3 m^{4}}{2} \cdot \frac{3 m^{2}}{2} \cdot \operatorname{diam} \mathbb{M},
$$

where the small unspecified constant is due to the mapping from the sphere to the rectangle. This implies the claimed bound.

The Koksma-Hlawka Theorem applies if the variation is bounded, which in our case holds for the Harman variation. We therefore mention the main result in [14], which assumes a bounded, measurable function $F:[0,1]^{s} \rightarrow \mathbb{R}$ for which $\operatorname{Har}(F)$ is bounded from above by a constant that does not depend on $N$ :

$$
\operatorname{Err}(N, X) \leq \operatorname{Dis}_{\mathcal{K}}(N, X) \cdot \operatorname{Har}(F)
$$

The Variation Lemma together with (18) for $s=2$ now imply a bound on the error in terms of the Harman variation.

Corollary. Let $\mathbb{M}$ and $F$ be as in the Variation Lemma. Then for every $N>N_{0}(2, \varepsilon)$, we have

$$
\operatorname{Err}(N, X) \leq \frac{\text { const } \cdot N^{\varepsilon}}{N^{2 / 3}} \cdot m^{6} \operatorname{diam} \mathbb{M}
$$

\section{Persistence and Stability}

In this section, we modify the length estimation formulas of Section 3 to get stable estimates for tube-like shapes. We begin with the introduction of persistent homology, which is instrumental to achieving this goal. We refer to [11] for more details about this concept.

Persistent homology. Let $\mathbb{M}$ be a compact body in $\mathbb{R}^{3}$, perhaps a tube or a tube-like shape, as before. Fixing a direction $u \in \mathbb{S}^{2}$, we define the height function, $f: \mathbb{M} \rightarrow \mathbb{R}$, by mapping each point $x$ to $f(x)=\langle x, u\rangle$. It is the signed distance from the plane with normal vector $u$ that passes through the origin. This function offers a convenient way to study the intersections of $\mathbb{M}$ with planes normal to $u$. Indeed, the level set of $f$ at $\alpha \in \mathbb{R}$, defined as $f^{-1}(\alpha)$, is the intersection of $\mathbb{M}$ with the plane of points $\langle x, u\rangle=\alpha$. Instead of looking at the sequence of level sets, we take an indirect approach and consider the sublevel sets, $\mathbb{M}_{\alpha}=f^{-1}(-\infty, \alpha]$, and the superlevel sets, $\mathbb{M}^{\alpha}=f^{-1}[\alpha, \infty)$. The reason is the containment relation, which allows us to relate the homology groups with one another.

We are now more specific. Excluding pathological cases, $f$ has only a finite number of homological critical values, $c_{1}$ to $c_{n}$. Choosing interleaving homological regular values, 
$\alpha_{0}<c_{1}<\alpha_{1}<\ldots<c_{n}<\alpha_{n}$, we consider the (absolute) homology groups of the sublevel sets, $H_{i}=\mathrm{H}\left(\mathbb{M}_{\alpha_{i}}\right)$, and the relative homology groups of $\mathbb{M}$ and the superlevel sets, $H_{2 n-i}=\mathrm{H}\left(\mathbb{M}, \mathbb{M}^{\alpha_{i}}\right)$. Here, $\mathrm{H}$ is the functor that maps a space or a pair of spaces to the direct sum of the homology groups of all dimensions. With this convention, we can simplify the common notation for homology, which would otherwise give the dimension in the subscript. Note that $H_{n}$ is defined twice, as the absolute homology of the sublevel set for $\alpha_{n}$, and the relative homology of $\mathbb{M}$ and the superlevel set for $\alpha_{n}$. Since $\mathbb{M}$ is compact, $\alpha_{n}$ is necessarily larger than the maximum height value, so $\mathbb{M}_{\alpha_{n}}=\mathbb{M}$ and $\mathbb{M}^{\alpha_{n}}=\emptyset$. It follows that the two groups are indeed the same. Similarly, $\mathbb{M}_{\alpha_{0}}=\emptyset$ and therefore $H_{0}=0$ as well as $\mathbb{M}^{\alpha_{0}}=\mathbb{M}$ and therefore $H_{2 n}=0$. Writing the groups in sequence, with the homomorphism induced by inclusions between them, we get

$$
0 \rightarrow \ldots \rightarrow H_{i-1} \rightarrow H_{i} \rightarrow \ldots \rightarrow H_{j-1} \rightarrow H_{j} \rightarrow \ldots \rightarrow 0
$$

where $0<i<j \leq 2 n$. A class $\gamma \in H_{i}$ is born at $H_{i}$ and dies entering $H_{j}$ if (i) $\gamma$ is not in the image of $H_{i-1}$ in $H_{i}$, and (ii) the image of $\gamma$ is not contained in the image of $H_{i-1}$ in $H_{j-1}$, but it is contained in the image of $H_{i-1}$ in $H_{j}$. By definition of homology groups, an entire coset of classes is born and dies together with $\gamma$. Note that the indices are chosen such that the homological critical value right before $H_{i}$ is $c_{i}$, for $1 \leq i \leq n$, and $c_{2 n-i+1}$, for $n<i \leq 2 n$. We therefore represent the coset born at $H_{i}$ and dying entering $H_{j}$ by the birth-death pair of homological critical values, $\left(\alpha_{b}, \alpha_{d}\right)$, where $\alpha_{b}=c_{i}$ or $c_{2 n-i+1}$ and $\alpha_{d}=c_{j}$ or $c_{2 n-j+1}$, depending on whether $i$ and $j$ are smaller than or equal to $n$ or larger than $n$. We call the absolute difference between these values, $\operatorname{pers}(\gamma)=\left|\alpha_{d}-\alpha_{b}\right|$, the persistence of $\gamma$, or of its birth-death pair.

Diagrams and moments. Each birth-death pair has a $d i$ mension, which it inherits from the homology classes it represents. Traditionally, the collection ${ }^{1}$ of $p$-dimensional birthdeath pairs is referred to as the $p$-dimensional persistence diagram of the function, denoted as $\operatorname{Dgm}_{p}(f)$. It describes the evolution of the $p$-dimensional homology of the sequence of sublevel and superlevel sets; see e.g. [11]. In this paper, we are interested in the level sets, whose Betti numbers can also be derived from the birth-death pairs, but now we need to consider two persistence diagrams at a time. We therefore distinguish the pairs for which the birth value is smaller than the death value from the others. Specifically, we define $\operatorname{Up}_{p}(f)$ as the multi-set of pairs in $\operatorname{Dgm}_{p}(f)$ for which $\alpha_{b} \leq \alpha_{d}$, and we define $\operatorname{Dn}_{p}(f)$ as the multi-set of pairs with $\alpha_{d} \leq \alpha_{b}$. The following result can be found in [3].

\footnotetext{
${ }^{1}$ More precisely, $\operatorname{Dgm}_{p}(f)$ is a multi-set of pairs since the homology may gain and lose more than one rank when $\alpha$ passes a homological critical value. For each such rank, we have a pair containing this critical value as one of its components. Nevertheless, we will use common terminology for sets to denote operations like containment and union. For an appropriately defined generic function (e.g. a Morse function on a manifold), such coincidences between changes in the homology do not happen, but there are no compelling reasons for us to restrict the functions this severely.
}

Level Set Lemma. Let $\alpha$ be a homological regular value of $f$. Then the $p$-th Betti number of $f^{-1}(\alpha)$ is equal to the number of $p$-dimensional birth-death pairs with $\alpha_{b}<\alpha<\alpha_{d}$ plus the number of $(p+1)$-dimensional birth-death pairs with $\alpha_{d}<\alpha<\alpha_{b}$.

All pairs of the first kind are in $\operatorname{Up}_{p}(f)$, while all pairs of the second kind are in $\operatorname{Dn}_{p+1}(f)$. It is now easy to integrate the $p$-th Betti number of $f^{-1}(\alpha)$, over all values $\alpha$. Indeed, each pair in $\mathrm{Up}_{p}(f)$ contributes $\alpha_{d}-\alpha_{b}$ to this integral, and each pair in $\operatorname{Dn}_{p+1}(f)$ contributes $\alpha_{b}-\alpha_{d}$. We therefore define

$$
B_{p}^{k}(f)=\sum_{A \in \cup_{p}(f)} \operatorname{pers}(A)^{k}+\sum_{A \in \operatorname{Dn}_{p+1}(f)} \operatorname{pers}(A)^{k},
$$

calling it the $k$-th $p$-dimensional persistence moment of the family of level sets. ${ }^{2}$ The first moment, $B_{p}^{1}(f)$, is the integral of $p$-dimensional Betti numbers mentioned earlier. We now apply these concepts to the second Quermassintegral, which for a compact body with smoothly embedded boundary in $\mathbb{R}^{3}$ is equal to the total mean curvature of this surface. As already observed in [5], this Quermassintegral can be expressed in terms of birth-death pairs and their persistence. The connection is formed by the Level Set Lemma and the first persistence moment. All we still need is to integrate over all directions $u$ of the unit sphere. Writing $f_{u}$ for the corresponding height function, we get

$$
\begin{aligned}
Q_{2}(\mathbb{M}) & =\frac{1}{2} \int_{u \in \mathbb{S}^{2}} \int_{\alpha=-\infty}^{\infty} \chi\left(f_{u}^{-1}(\alpha)\right) \mathrm{d} \alpha \mathrm{d} u \\
& =\frac{1}{2} \int_{u \in \mathbb{S}^{2}}\left(\sum_{p=0}^{2}(-1)^{p} B_{p}^{1}\left(f_{u}\right)\right) \mathrm{d} u .
\end{aligned}
$$

In words, the 2-nd Quermassintegral is half the integral, over all directions, of the alternating sum of first persistence moments.

The first moments are not stable, and small perturbations of $\mathbb{M}$ can cause large differences. To illustrate this, we recall that $Q_{2}=Q_{2}(\mathbb{M})$ is the total mean curvature of the boundary of $\mathbb{M}$. Suppose we add a small bump, of the kind illustrated in Figure 6 and discussed in Appendix A. If the radius of the defining circles is $\varepsilon$, then the bump contributes $(4-\pi) \pi \varepsilon$ to the mean curvature. Suppose we have space to add $\ell$ bumps, denoting the new body by $\mathbb{M}_{[\varepsilon]}$. The Hausdorff distance between $\mathbb{M}$ and $\mathbb{M}_{[\varepsilon]}$ is only about $2 \varepsilon$, while the total mean curvature of $\mathbb{M}_{[\varepsilon]}$ is about $\ell(4-\pi) \pi \varepsilon$ larger than that of $\mathbb{M}$. If we shrink the bumps to radius $\frac{\varepsilon}{2}$, we get a body $\mathbb{M}_{[\varepsilon / 2]}$ with about $4 \ell$ smaller bumps and Hausdorff distance about $\varepsilon$ from $\mathbb{M}$. The total mean curvature of $\mathbb{M}_{[\varepsilon / 2]}$ is about $2 \ell(4-\pi) \pi \varepsilon$ larger than that of $\mathbb{M}$. Continuing this way, we get a sequence of bodies whose Hausdorff distance to $\mathbb{M}$ goes to zero, while the total mean curvature goes to infinity.

\footnotetext{
${ }^{2} \mathrm{We}$ note that there is a difference between the persistence moments defined here for level sets, and the persistence moments in [7] defined for sublevel sets. While the difference is important for our purposes, it is not substantial. In particular, the $k$-th moment in [7] is $\operatorname{Pers}_{k}(f)=$ $\sum_{A \in \operatorname{Dgm}(f)} \operatorname{pers}(A)^{k}$. Being the sum of the $k$-th powers over all dimensions, it clearly bounds $B_{p}^{k}(f)$ from above. It follows that the Moment Lemma in [7] also applies to $B_{p}^{k}(f)$; see (36) and (37) below.
} 
Small and large persistence. The above example suggests that the 1-st persistence moments are instable because small perturbations cause the diagram to gain or lose many birthdeath pairs with small persistence, and not because large persistence pairs change. This is a general phenomenon, which motivates our next step. We distinguish between birth-death pairs with persistence less than $r_{0}$, calling them small, and birth-death pairs with persistence at least $r_{0}$, calling them large. Accordingly, we define

$$
\begin{aligned}
& B_{p}^{k}\left(f, r_{0}^{-}\right)=\sum_{\operatorname{pers}(A)<r_{0}} \operatorname{pers}(A)^{k}, \\
& B_{p}^{k}\left(f, r_{0}^{+}\right)=\sum_{\operatorname{pers}(A) \geq r_{0}} \operatorname{pers}(A)^{k},
\end{aligned}
$$

where the sum is over all birth-death pairs $A$ in $\operatorname{Up}_{p}(f)$ and in $\operatorname{Dn}_{p+1}(f)$, as in (28). Note that $B_{p}^{k}(f)$ is the sum of the two. Using $r_{0}$ as a parameter of scale, we can now separate the contributions of features whose scale is smaller than that parameter. We do this by damping the contribution of a small birth-death pair $A$ by pers $(A)^{k-1} / r_{0}^{k-1}$, which for $k>1$ is necessarily less than 1. Equivalently, the contribution of a small birth-death pair is $1 / r_{0}^{k-1}$ times the $k$-th power of the persistence. For technical reasons that will become clear shortly, we choose $k=C=4+\delta$, in which $\delta$ is a small positive real number. With this, we modify (30) by defining

$$
\begin{aligned}
& X_{p}^{C}(f)=B_{p}^{1}\left(f, r_{0}^{+}\right)+\frac{1}{r_{0}^{C-1}} \cdot B_{p}^{C}\left(f, r_{0}^{-}\right), \\
& \bar{Q}_{2}(\mathbb{M})=\frac{1}{2} \int_{u \in \mathbb{S}^{2}}\left(\sum_{p=0}^{2}(-1)^{p} X_{p}^{C}\left(f_{u}\right)\right) \mathrm{d} u,
\end{aligned}
$$

calling $\bar{Q}_{2}=\bar{Q}_{2}(\mathbb{M})$ the stabilized mean curvature of $\mathbb{M}$ at scale $r_{0}$. Recall that the contribution of a large birth-death pair to $X_{p}^{C}(f)$ is the same as to $B_{p}^{1}(f)$, while the contribution of a small birth-death pair to $X_{p}^{C}(f)$ is smaller than to $B_{p}^{1}(f)$. This implies $X_{p}^{C}(f) \leq B_{p}^{1}(f)$, but since $Q_{2}$ and $\bar{Q}_{2}$ are both alternating sums of moments, this does not imply any particular relation between the unstabilized and the stabilized mean curvatures of $\mathbb{M}$. Recall that $\mathbb{M}_{[\varepsilon]}$ is $\mathbb{M}$ modified by attaching bumps of size $\varepsilon$. As argued above, $Q_{2}\left(\mathbb{M}_{[\varepsilon]}\right)$ goes to infinity as $\varepsilon$ approaches zero. In contrast, $\bar{Q}_{2}\left(\mathbb{M}_{[\varepsilon]}\right)$ barely changes. The remainder of this section analyzes to what extent this is true in general.

Meshing tubes. The reason for the particular choice of power is the complexity of triangulating a tube, as we now explain. We will make heavy use of the results described in [7], and minimize repetition by appealing within footnotes to specific arguments in that paper. Let $\mathbb{M} \subseteq \mathbb{R}^{3}$ be obtained by uniformly thickening a closed curve of length $L$, and let $r_{0}$ be the radius of an orthogonal cross-section. Here we assume that the curve has no forks, although the analysis below would allow for a small number of forks. A triangulation of $\mathbb{M}$ is a simplicial complex together with a homeomorphism from its underlying space to $\mathbb{M}$. Its mesh is the maximum distance between two points in $\mathbb{M}$ whose preimages belong to a common simplex. Let $N(r)$ be the minimum number of simplices needed for a triangulation of $\mathbb{M}$ with mesh $r$ or less. This function follows different regimes for radii less than and greater equal than $r_{0}$ :

$$
N(r)= \begin{cases}\text { const } \cdot L r_{0}^{2} / r^{3} & \text { if } r<r_{0} \\ \text { const } \cdot L / r & \text { if } r \geq r_{0}\end{cases}
$$

Both estimates follow from simple volume arguments. Let now $f: \mathbb{M} \rightarrow \mathbb{R}$ be the height function on $\mathbb{M}$ in some direction, as before, and note that it is 1-Lipschitz, which means that $|f(x)-f(y)| \leq\|x-y\|$ for all points $x, y \in \mathbb{M}$. For such a function, the Moment Lemma in [7] implies

$$
\begin{aligned}
& B_{p}^{1+\delta}\left(f, r_{0}^{+}\right) \leq \text {const } \cdot L^{1+\delta}, \\
& B_{p}^{3+\delta}\left(f, r^{+}\right) \leq \text {const } \cdot L^{1+\delta} r_{0}^{2} .
\end{aligned}
$$

Importantly, the first and stronger inequality holds for $r_{0}$, while the second and weaker inequality holds for general positive radii. ${ }^{3}$ We make use of both inequalities, but for different purposes. From (36), we get an upper bound on the number of large birth-death pairs:

$$
B_{p}^{0}\left(f, r_{0}^{+}\right) \leq \text {const } \cdot L^{1+\delta} / r_{0}^{1+\delta},
$$

simply because every counted birth-death pair contributes at least $r_{0}^{1+\delta}$ to the $(1+\delta)$-th moment. From (37), we get a statement of stability for the $(4+\delta)$-th moment:

$$
\left|B_{p}^{4+\delta}(f)-B_{p}^{4+\delta}(g)\right| \leq \text { const } \cdot L^{1+\delta} r_{0}^{2} \cdot\|f-g\|_{\infty},
$$

where $f$ and $g$ are two 1-Lipschitz functions on $\mathbb{M}$. ${ }^{4}$ Here we note that (39) also holds if $g$ is not Lipschitz but still satisfies (37); see the proof of the Total Persistence Theorem in [7].

Stability analysis. We now return to proving that the stabilized mean curvature defined in (34) is indeed a stable quantity. By this we mean that if $\mathbb{M}$ is perturbed by a small amount, then the estimate changes only by a small amount. Specifically, we allow for a homeomorphism $\mu: \mathbb{M} \rightarrow \mathbb{M}^{\prime}$ and measure the distortion by taking the maximum distance between corresponding points. To suitably limit the class of perturbations, we introduce $g: \mathbb{M} \rightarrow \mathbb{R}$ defined by mapping $x \in \mathbb{M}$ to the height of $\mu(x) \in \mathbb{M}^{\prime}$, and we require that $g$ be Lipschitz. While this is perhaps overly conservative, it is easy to see how to relax the requirement to a more technical condition more closely related to the proof of the upcoming result. Note that the persistence diagrams of $g$ and of the height function on $\mathbb{M}^{\prime}$ are the same. We can therefore use

\footnotetext{
${ }^{3}$ Indeed, for large persistence pairs, we have $M=1$, in the terminology of [7, p. 134], and therefore $A, B \leq$ const $\cdot L^{1+\delta}$, again in that terminology. For small persistence pairs, we have $M=3$ and therefore $A, B \leq$ const . $L^{1+\delta} r_{0}^{2}$.

${ }^{4}$ To see this, we note that $\mathbb{M}$ has bounded degree- $(3+\delta)$ total persistence in the terminology of [7, p. 136]. The Total Persistence Stability Theorem of that paper now implies that for $k=4+\delta$, the difference between the moments is at most const $\cdot L^{1+\delta} r_{0}^{2}$ times the maximum difference between the functions.
} 
$g$ as a proxy in the comparison with $f$. Also note that the difference between the functions is bounded from above by the distortion: $\|f-g\|_{\infty} \leq \max _{x \in \mathbb{M}}\|x-\mu(x)\|$.

Dampened Stability Theorem for Tubes. Let $\mathbb{M}$ be a tube of radius $r_{0}$ in $\mathbb{R}^{3}$, let $f, g: \mathbb{M} \rightarrow \mathbb{R}$ be Lipschitz functions on the tube with $\|f-g\|_{\infty}<r_{0} / 4$, and set $C=4+\delta$. Then for every dimension $0 \leq p \leq 2$, every direction $u \in \mathbb{S}^{2}$, and every $\delta>0$, we have

$$
\left|X_{p}^{C}(f)-X_{p}^{C}(g)\right| \leq \text { const } \cdot \frac{L^{1+\delta}}{r_{0}^{1+\delta}} \cdot\|f-g\|_{\infty} .
$$

Proof. We compare $X_{p}^{C}(f)$ with $X_{p}^{C}(g)$ indirectly, by comparing both with another quantity. To motivate that quantity, we recall the Stability Theorem of Persistence proved in [6], which implies that there is a bijection between the birthdeath pairs $A$ of $f$ and $A^{\prime}$ of $g$ such that $\left|\operatorname{pers}(A)-\operatorname{pers}\left(A^{\prime}\right)\right| \leq$ $2 \varepsilon$, in which we write $\varepsilon=\|f-g\|_{\infty}$. With the same notation, we now define

$$
Y_{p}^{C}(f, g)=\sum_{\operatorname{pers}(A) \geq r_{0}} \operatorname{pers}\left(A^{\prime}\right)+\frac{1}{r_{0}^{C-1}} \cdot \sum_{\operatorname{pers}(A)<r_{0}} \operatorname{pers}\left(A^{\prime}\right)^{C},
$$

where the sum is over all birth-death pairs $A$ of $f$, and $A^{\prime}$ is the image of $A$ under the mentioned bijection. The difference to the first moment, $\Delta_{1}=\left|X_{p}^{C}(f)-Y_{p}^{C}(f, g)\right|$, is small because of the mentioned theorem:

$$
\begin{aligned}
\Delta_{1} \leq & \sum_{\operatorname{pers}(A) \geq r_{0}}\left|\operatorname{pers}(A)-\operatorname{pers}\left(A^{\prime}\right)\right| \\
& +\frac{1}{r_{0}^{C-1}} \cdot \sum_{\operatorname{pers}(A)<r_{0}}\left|\operatorname{pers}(A)^{C}-\operatorname{pers}\left(A^{\prime}\right)^{C}\right| \\
\leq & \text { const } \cdot \frac{L^{1+\delta}}{r_{0}^{1+\delta}} \cdot \varepsilon,
\end{aligned}
$$

in which we go from (42) to (43) by applying (38) to the first sum and (39) to the second sum. Here it is important that $C=$ $4+\delta$ and not smaller, else (39) would not have a proof. The difference to the second moment, $\Delta_{2}=\left|X_{p}^{C}(g)-Y_{p}^{C}(f, g)\right|$, is small because only the points $A^{\prime}$ that correspond to points $A$ with persistence between $r_{0} \pm 2 \varepsilon$ are possibly misclassified:

$$
\begin{aligned}
\Delta_{2} & \leq \sum_{\left|\operatorname{pers}(A)-r_{0}\right| \leq 2 \varepsilon}\left|\operatorname{pers}\left(A^{\prime}\right)-\frac{1}{r_{0}^{C-1}} \operatorname{pers}\left(A^{\prime}\right)^{C}\right| \\
& \leq \operatorname{const} \cdot \frac{L^{1+\delta}}{r_{0}^{1+\delta}} \cdot \varepsilon,
\end{aligned}
$$

where we get the final inequality by multiplying the number of points with the maximum possible difference. We get the bound on the number of points from (38), after substituting $r_{0}-2 \varepsilon>r_{0} / 2$ for $r_{0}$ and absorbing the difference into the constant. To bound the maximum possible difference, we use pers $\left(A^{\prime}\right) \leq r_{0}+4 \varepsilon$. Writing $\varepsilon_{0}=4 \varepsilon / r_{0}$, the difference contributed to (44) is

$$
\begin{aligned}
\Delta^{\prime} & =\operatorname{pers}\left(A^{\prime}\right)\left[\frac{\operatorname{pers}\left(A^{\prime}\right)^{C-1}}{r_{0}^{C-1}}-1\right] \\
& \leq r_{0}\left(1+\varepsilon_{0}\right)\left[\left(1+\varepsilon_{0}\right)^{C-1}-1\right],
\end{aligned}
$$

which can be rewritten as $\Delta^{\prime} \leq r_{0}\left[\left(1+\varepsilon_{0}\right)^{C}-\left(1+\varepsilon_{0}\right)\right]$. For $\varepsilon_{0}=0,1$, we get 0 and $r_{0}\left[2^{C}-2\right]$ on the right hand side. By assumption, the value of $\varepsilon_{0}$ lies between 0 and 1 , and by convexity of the function $x^{C}$ in this interval, we get

$$
\Delta^{\prime}<r_{0} \varepsilon_{0}\left(2^{C}-2\right)=\varepsilon\left(2^{C+2}-8\right)
$$

Absorbing the factor into the constant, we get (45). Finally, we get $\left|X_{p}^{C}(f)-X_{p}^{C}(g)\right| \leq \Delta_{1}+\Delta_{2}$, which implies the claimed inequality.

The Dampened Stability Theorem implies a similar upper bound for the difference between $\bar{Q}_{2}(\mathbb{M})$ and $\bar{Q}_{2}\left(\mathbb{M}^{\prime}\right)$, which implies that $\bar{Q}_{2}$ is a stable estimate of the total mean curvature. It is important to realize that this statement can only be true for a suitably limited class of perturbations $\mathbb{M}^{\prime}$ of $\mathbb{M}$. The crucial property here is that the height function on $\mathbb{M}^{\prime}$ pulled back to $\mathbb{M}$ satisfy (37). For this inequality to hold, it is sufficient but not necessary that the pulled back height function be Lipschitz. It would be interesting to further sharpen the description of the class of perturbations for which the Dampened Stability Theorem holds.

\section{Computational Experiments}

In this section, we describe experimental results for the algorithms implementing the mathematical formulas developed in the preceding sections. We test accuracy as well as stability on small datasets, for which the answers are known, and investigate speed of convergence on root system data.

Algorithms. We use three different algorithms to compute or approximate the total mean curvature of the boundary of a polytope $\mathbb{M}$ in $\mathbb{R}^{3}$, and to estimate the length of $\mathbb{M}$.

- Discrete Mean Curvature (DMC): we compute the total mean curvature as half the sum over all boundary edges of the length times the angle between the two adjacent face normals; see e.g. [8].

- Plane Sampling (PS): we approximate the total mean curvature by summing up the Euler characteristics of the intersections between $\mathbb{M}$ and planes sampled in $\mathbb{R}^{3}$.

- Direction Sampling (DS): we approximate the total mean curvature by summing up the alternating persistence moments of height functions defined by sampled directions on the 2-sphere.

The result of the DMC Algorithm is the total mean curvature of $\mathbb{M}$ up to machine precision, which we use as the baseline for comparisons. For shapes defined as the union of integer cubes, the computations are particularly easy. Every convex edge belongs to one cube and contributes $\frac{\pi}{2}$ to the total mean curvature, while every reflex edge belongs to three cubes and contributes $-\frac{\pi}{2}$. Every edge that belongs to two cubes that do not share a face is considered a pair of reflex 
edges that contributes $-\pi$. This is consistent with interpreting $\mathbb{M}$ as the union of closed cubes. All other edges contribute zero. The result of the PS Algorithm converges to the total mean curvature, and we get an impression of the speed of convergence from a comparison with the precise measurement. The basic version of the DS Algorithm is a reformulation of the PS Algorithm, but it offers the opportunity to filter out low-persistence contributions, thus stabilizing the length estimate.

Plane sampling. We assume a polytope $\mathbb{M}$ with a finite number of vertices in $\mathbb{R}^{3}$. Let $\rho$ be the maximum distance of any of these vertices from the origin. For a curve with endpoint or forks, we also need an estimate of the tube radius, which we denote by $r_{0}$. We use a global estimate as there is not much difference between the total mean curvature of a uniformly and a non-uniformly thick tube, provided the radius function has small variation, which we assume.

STEP 1. Construct a collection of planes, $\mathcal{P}$, such that their parameter triplets, $(\varrho, a, \varphi)$, form a point set that is uniformly distributed in $[-\rho, \rho] \times[0,1] \times[0,2 \pi]$. Write $\# \mathcal{P}$ for the number of planes.

STEP 2. Intersect each plane $P$ in $\mathcal{P}$ with $\mathbb{M}$, and compute the Euler characteristic, $\chi(\mathbb{M} \cap P)$, by counting the components and holes of the intersection.

STEP 3. Compute an approximation of the Quermassintegral by multiplying the volume of the domain with the average Euler characteristic:

$$
\tilde{Q}_{2}=\frac{4 \rho \pi}{\# \mathcal{P}} \sum_{P \in \mathcal{P}} \chi(\mathbb{M} \cap P) .
$$

Finally, return the length estimate according to the Equations (1) or (2).

We distinguish between two implementations of this algorithm: rPS samples the planes randomly, and qPS does quasi-random sampling using $(t, s)$-sequences as introduced by Niederreiter [22]; see also [1, 10]. We expect that the convergence to the total mean curvature is slower for random sampling, and this is what we observe.

Direction sampling. Different from the PS Algorithm, we now sample directions, which we represent by points $(a, \varphi)$ in $[0,1] \times[0,2 \pi]$. For the stabilized formula, we need again the estimate of the tube radius, $r_{0}$.

STEP 1. Construct a collection of directions, $\mathcal{U}$, such that their parameter pairs, $(a, \varphi)$, are uniformly distributed in $[0,1] \times[0,2 \pi]$. Write $\# \mathcal{U}$ for this number.

STEP 2. For each direction $u \in \mathcal{U}$, compute the alternating sum of persistence moments, as given in (30).
STEP 3. Compute the approximation of the Quermassintegral by multiplying the area of the domain with the average alternating sum of persistence moments:

$$
\tilde{Q}_{2}=\frac{2 \pi}{\# \mathcal{U}} \sum_{u \in \mathcal{U}} \sum_{p=0}^{2}(-1)^{p} B_{p}^{1}\left(f_{u}\right) .
$$

Finally, return the length estimate according to Equations (1) or (2).

Similar to plane sampling, we distinguish between rDS, which samples directions randomly, and qDS, which uses quasi-random sampling. In addition, we consider versions $\mathrm{DS}_{1}$ and $\mathrm{DS}_{C}$ that differ from each other in the damping of low persistence contributions. In particular, $\mathrm{qDS}_{1}$ an $\mathrm{rDS}_{1}$, given in (50), are consistent with the basic relationship between length and total mean curvature, while $\mathrm{rDS}_{C}$ and $\mathrm{qDS}_{C}$ substitute $X_{p}^{C}\left(f_{u}\right)$ for $B_{p}^{1}\left(f_{u}\right)$, which is the stabilized total mean curvature given in (34).

Stability. We test the stability of our algorithm on a set of four datasets, three of which are illustrated in Figure 2. In its original form shown at the top, Cylinder consists of

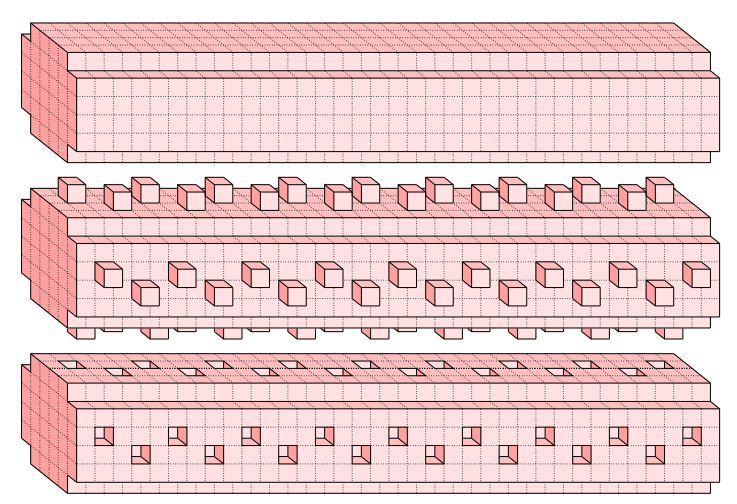

Figure 2: Three of the four small datasets used to test the stability of our algorithm: Cylinder at the top and its noisy versions, BumpyC and Holeyc, below.

35 disk-like sections, each consisting of 32 voxels. There are 328 convex and 140 reflex edges, giving a total mean curvature of $328 \frac{\pi}{4}-140 \frac{\pi}{4}=47 \pi$. If we plug this value into the length formula (2), setting the number of forks to $k=0$, the number of tips to $\ell=2$, and the tube radius to $r_{0}=3.0$, we get

$$
L=\left(47 \pi-4 \pi r_{0}\right) / \pi=35.0,
$$

which is right on target. The achieved accuracy is however a coincidence caused by the agreement of the discrete mean curvature of the model with the total mean curvature of the round cylinder of same length and radius and closed off with half-spheres at the two ends. Two noisy versions of the model, BumpyC and HoleyC in Figure 2, are obtained by adding and removing 68 voxels from Cylinder. Table 2 
shows the total mean curvature computed by our algorithm. For BumpyC, it is considerably larger, and for HoleyC, it is considerably smaller than for $\mathrm{Cy}$ linder. In contrast, the stabilized estimates computed by $\mathrm{qDS}_{C}$ are not very different from each other.

\begin{tabular}{l||r|rrrr} 
Datasets & \#voxels & DMC & \multicolumn{1}{c}{ qPS } & qDS $_{1}$ & qDS $_{C}$ \\
\hline \hline Cylinder & 1,120 & $47 \pi$ & $45.38 \pi$ & $46.96 \pi$ & $45.78 \pi$ \\
\hline BumpyC & 1,188 & $115 \pi$ & $120.93 \pi$ & $115.19 \pi$ & $47.14 \pi$ \\
\hline HoleyC & 1,052 & $-21 \pi$ & $-14.95 \pi$ & $-21.27 \pi$ & $44.90 \pi$ \\
\hline LongC & 1,154 & $63 \pi$ & $61.72 \pi$ & $62.93 \pi$ & $58.47 \pi$
\end{tabular}

Table 2: The size and total mean curvature of the four cylinder datasets, as computed by four implementations of our algorithms. The last three algorithms approximate the result by sampling 300 planes and directions, respectively. The last algorithm requires the estimated tube radius as an additional parameter, which is set to $r_{0}=3.0$ for the three models in Figure 2 and to $r_{0}=2.27$ for LongC. The power used to stabilize the length estimate is set to $C=4.1$.

To complement these results, we compute the total mean curvature of LongC, which is obtained by adding a tail of length 16 to Cylinder on the left. The tail has a thick part, consisting of 6 sections of four voxels each, and a thin part, which is a row of 10 voxels. In total, we add $24+10=34$ voxels to Cylinder, as shown in the first column of Table 2. This modification of the original model cannot be considered noise, and this is indeed born out by our computations, which show that the total mean curvature estimated by qDS is barely smaller than those of the other three algorithms. The fact that the stabilization does not change the estimate by much is indicative of the tail being a feature of LongC and not a noisy artifact. For the computations, we use a weighted average of the radii along the three pieces as the estimated radius, namely $\frac{35}{51} \cdot 3.0$ plus $\frac{6}{51} \cdot 1.0$ plus $\frac{10}{51} \cdot 0.5$, which is roughly $r_{0}=2.27$. Using $\mathrm{qDS}_{C}$ and Equation (2) with $k=0$ forks and $\ell=2$ tips, we get

$$
L=(58.47 \pi-4 \pi \cdot 2.27) / \pi=49.39 \ldots
$$

as the stabilized length estimate, which is slightly less than 51. Comparing this with the stabilized length estimate for Cylinder, which is $(45.78 \pi-4 \pi \cdot 3.0)=33.78 \ldots$, we note that the increment is close to 16 , which is what it should be.

Fork compensation. To illustrate the compensation for forks in our length formula as described in Section 2, we create ForkC by adding two side branches of length 8 each to Cylinder; see Figure 3. The DMC algorithm computes the total mean curvature of ForkC as $63 \pi$. The number of forks is $k=2$, the number of tips is $\ell=4$, and for the radius we set again $r_{0}=3.0$. Plugging these values into (2), we get

$$
L=\left[63 \pi+\left(4+C_{0}\right) 2 r_{0}-8 \pi r_{0}\right] / \pi
$$

which for $C_{0}=3.459 \ldots$ gives $L=53.245 \ldots$. We compare this with the length of 57, which we get by adding the lengths of the three curves that form the (connected) axis of the dataset. Indeed, the length of the curve that gives

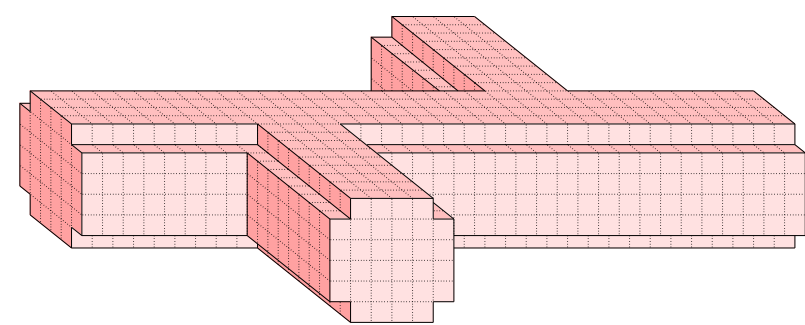

Figure 3: ForkC is obtained from Cylinder by adding two side branches. It is a tube-like shape with two forks and four tips.

the main branch is 35 , and for each side branch, we get $8+r_{0}=11$. A detailed analysis of the contributions to our length estimate shows that the main reason for the difference is the larger lost area in our model as compared to the round cylinder. Indeed, we lose about $6 \pi$ in total mean curvature because of the lost area per fork, while we only compensate for $4 r_{0}=12.0$ of the loss. Multiplying with the number of forks and dividing by $\pi$ to get the length, gives a shortfall of $(12 \pi-24.0) / \pi=4.36 \ldots$ in compensation, which is only slightly more than the shortfall we notice. This overestimate can be explained by the fact that the (negative) mean curvature along the creases where the side branches meet the main branch is slightly smaller, in absolute value, than the compensated amount.

Convergence. We finally study the rate of convergence of our algorithms. As expected, it depends on the sampling method, with quasi-random sampling leading to faster convergence than random sampling. To illustrate this phenomenon, we run our algorithms on a 3-dimensional reconstruction of the root system of a rice plant; see Figure 4. As explained in [28], the root system is imaged from dif-
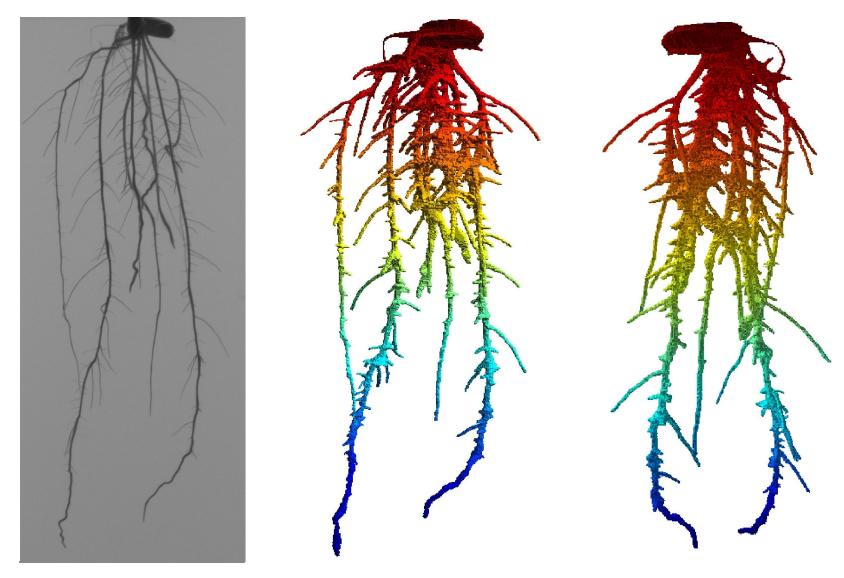

Figure 4: From left to right: a 2D gray-value image of a root system, and two views of the reconstructed $3 \mathrm{D}$ voxel model.

ferent angles, and the 2-dimensional images are processed to reconstruct a 3-dimensional voxel model. The biological interest in the model is the possibility to collect refined phenotype markers that help connecting the genotype with the 
phenotype of the root system; see e.g. [16]. The total length is an important such marker, but it is troubled by sensitivity to small shape variations, which have many sources. This is the main motivation behind our paper.

Returning to the topic at hand, we compute the (unstabilized) total mean curvature of the root system with rPS, $\mathrm{qPS}, \mathrm{rDS}_{1}$, and $\mathrm{qDS}_{1}$, for various numbers of sampled planes and directions. Figure 5 shows the results, including the total mean curvature as computed by the DMC Algorithm. The

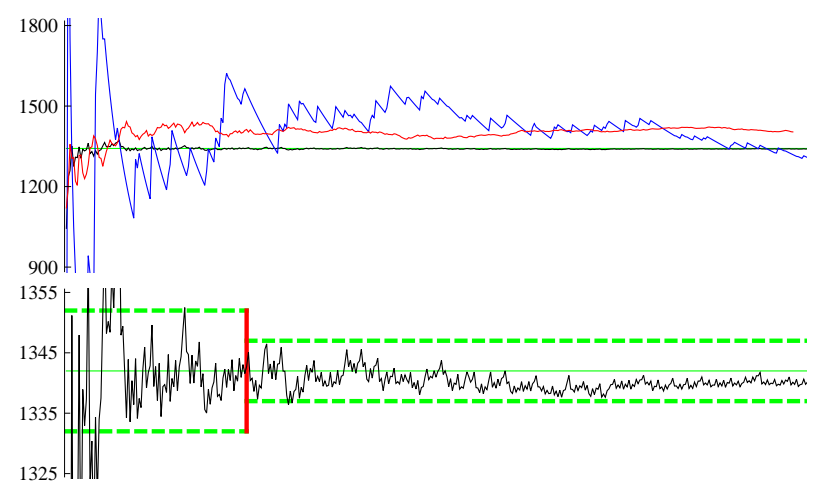

Figure 5: Comparison of the convergence of our algorithms: DMC (green), qPS (blue), $\mathrm{rDS}_{1}$ (red), and $\mathrm{qDS}_{1}$ (black). The horizontal axis counts the sampled entities, which are either planes or directions, and the vertical axis measures the total mean curvature. In the lower image, we scale up the vertical axis to show how the graph as computed by $\mathrm{qDS}_{1}$ converges to the baseline value computed by DMC. The red line marks 100 samples. The two corridors mark a deviation of $\pm 0.7 \%$ and $\pm 0.3 \%$ from the DMC value (green).

model of the root system consists of 10,933 voxels, and we sample up to 400 planes and directions to run our algorithms. Convergence is much faster for direction sampling, which is not surprising since the alternating sum of persistence moments of a single direction combines the information of all planes with that normal direction. We also observe that the convergence for quasi-random sampling is substantially faster than for random sampling. In Figure 5, this can be seen by comparing the curves for $\mathrm{rDS}_{1}$ and $\mathrm{qDS}_{1}$. We do not show the data for rPS because its convergence rate is substantially slower than that of qPS, to the extent that showing the curve would be detrimental to the figure. In contrast, $\mathrm{qDS}_{1}$ converges so fast that its graph is difficult to distinguish from the baseline obtained with the DMC algorithm. We therefore highlight the difference between these two graphs by exaggerating the vertical coordinate; see lower part of Figure 5.

\section{Discussion}

The main contribution of this paper is an algorithm for the length estimation of tube-like shapes that is stable under perturbations of the shape. This is the version $\mathrm{qDS}_{C}$ of the Direction Sampling Algorithm given in Section 5. Comparing this algorithm with others, we provide experimental evidence for its stability. Tube-like structures abound in the sciences, and our stable length estimation applies to most. We recall that the stabilized formula needs an estimate of the thickness, which makes sense for river networks, trees, blood vessels, lymph vessels, lung networks, dendrites, and more. There are other 1-dimensional networks that lack thickness, such as coastlines and district borders. Our formulas still apply, except that the interpretation of the parameter $r_{0}$ is the resolution of the estimate, rather than the thickness of the structure.

As usual, new insights come with new questions. Most interesting from a practical viewpoint is the extension of the methods to other measurements of shape, including

- the angle at which side-branches grow out of main stems in a root system;

- the symmetry of a natural network;

- the interaction between different networks, or between different portions of the same network.

Scale plays a role in all these questions, and describing a stable and meaningful measurement is the general challenge. On the mathematical side, it would be useful to extend the notion of Harman variation beyond convex sets. In particular, it might be possible to prove discrepancy bounds for hourglass bodies defined to relate to the body bounded by a hyperboloid like a convex body relates to a ball.

\section{Acknowledgements}

We would like to thank Olga Symonova and Michael Kerber for sharing their implementation of the persistence algorithm. Furthermore, we thank the reviewers for their careful reading of an earlier manuscript and a number of insightful comments which helped us to improve the paper.

\section{References}

[1] Ch. Aistleitner, J. S. Brauchart and J. Dick. Point sets on the sphere $\mathbb{S}^{2}$ with small spherical cap discrepancy. Discrete Comput. Geom., to appear.

[2] J. Beck. On the discrepancy of convex plane sets. Monatsh. Math. 105 (1988), 91-106.

[3] P. Bendich, H. Edelsbrunner, D. Morozov and A. Patel. Homology and robustness of level and interlevel sets. Homology, Homotopy, and Applications, to appear.

[4] H. Buum. A transformation for extracting new descriptors of shape. In Models for the Perception of Speech and Visual Form, 362-380, ed. W. Wathen-Dunn, MIT Press, Cambridge, Massachusetts, 1967.

[5] D. Cohen-Steiner and H. Edelsbrunner. Inequalities for the curvature of curves and surfaces. Found. Comput. Math. 7 (2007), 391404.

[6] D. Cohen-Steiner, H. Edelsbrunner and J. Harer. Stability of persistence diagrams. Discrete Comput. Geom. 37 (2007), 103-120.

[7] D. Cohen-Steiner, H. Edelsbrunner, J. Harer and Y. Mileyko. Lipschitz functions have $L_{p}$-stable persistence. Found. Comput. Math. 10 (2010), 127-139. 
[8] D. Cohen-Steiner and J.-M. Morvan. Restricted Delaunay triangulations and normal cycles. In "Proc. 19th Ann. Sympos. Comput. Geom., 2003", 312-321.

[9] L. De Floriani and M. Spagnuolo, eds. Shape Analysis and Structuring. Springer-Verlag, Berlin, Germany, 2008.

[10] J. Dick and F. Pillichshammer. Digital Nets and Sequences. Cambridge Univ. Press, Cambridge, England, 2010.

[11] H. Edelsbrunner and J. L. Harer. Computational Topology. An Introduction. Amer. Math. Soc., Providence, Rhode Island, 2010.

[12] H. Edelsbrunner, D. Letscher and A. J. Zomorodian. Topological persistence and simplification. Discrete Comput. Geom. 28 (2002), 511-533.

[13] A. Gray. Tubes. Addison-Wesley, Redwood City, California, 1990.

[14] G. Harman. Variations on the Koksma-Hlawka inequality. Unif. Distrib. Theory 5 (2010), 65-78.

[15] E. HLawKa. Funktionen von beschränkter Variation in der Theorie der Gleichverteilung. Ann. Math. Pura Appl. 54 (1961), 325-333.

[16] A. Iyer-Pascuzzi, O. Symonova, Y. Mileyko, Y. Hao, H. Belcher, J. HARER, J. S. WeItZ AND P. BeNFEY. Imaging and analysis platforms for automatic phenotyping and classification of plant root systems. Plant Physiol. 152 (2010), 1148-1157.

[17] J. F. Кокsмa. Een algemeene stelling inuit de theorie der gelijkmatige verdeeling modulo 1. Mathematica (Zutphen B) 11 (1942-43), 7-11.

[18] L. Kuipers and H. NiederReiter. Uniform Distribution of Sequences. Wiley, New York, New York, 1974.

[19] B. Mandelbrot. How long is the coast of Britain? Statistical selfsimilarity and fractional dimension. Science 156 (1967), 636-638.

[20] J.-M. Morvan. Generalized Curvatures. Springer-Verlag, Berlin, Germany, 2008.

[21] H. NiedERReiter. Methods for estimating discrepancy. In Applications of Number Theory to Numerical Analysis, 203-236, ed.: S. K. Zaremba, Academic Press, New York, 1972.

[22] H. Niederreiter. Point sets and sequences with small discrepancy. Monatsh. Math.104 (1987), 273-337.

[23] W. M. Schmidt. Irregularities of distribution IX. Acta Arith. XXVII (1975), 385-396.

[24] J. SteInER. Über parallele Flächen. Monatsber. Akad. Wiss. Berlin (1840), 114-118; also Werke, vol. 2 (1882), 171-178.

[25] W. Stute. Convergence rates for the isotrope discrepancy. Ann. Probab. 105 (1977), 91-106.

[26] H. WeYL. On the volume of tubes. Am. J. Math. 61 (1939), 461-472.

[27] S. K. Zaremba. Good lattice points in the sense of Hlawka and Monte Carlo integration. Monatsh. Math. 72 (1968), 264-269.

[28] Y. Zheng, S. Gu, H. Edelsbrunner, C. Tomasi and P. Benfey. Detailed reconstruction of 3D plant root shape. In "Proc. 13th Internat. Conf. Comput. Vision, 2011”, 2026-2033. 


\section{A Bumps}

In this appendix, we study the unit bump, which we define by rotating two quarter circles of unit radius, as illustrated in Figure 6. It consists of a hemi-sphere, for $0 \leq x \leq 1$, and a quarter torus, for $-1 \leq x \leq 0$. Our main result is that the total mean curvature of the unit bump is

$$
\text { Mean }_{\text {bump }}=(4-\pi) \pi .
$$

Note that scaling the bump by a factor of $\varepsilon$ changes its area by a factor of $\varepsilon^{2}$ and its total mean curvature by a factor of $\varepsilon$.

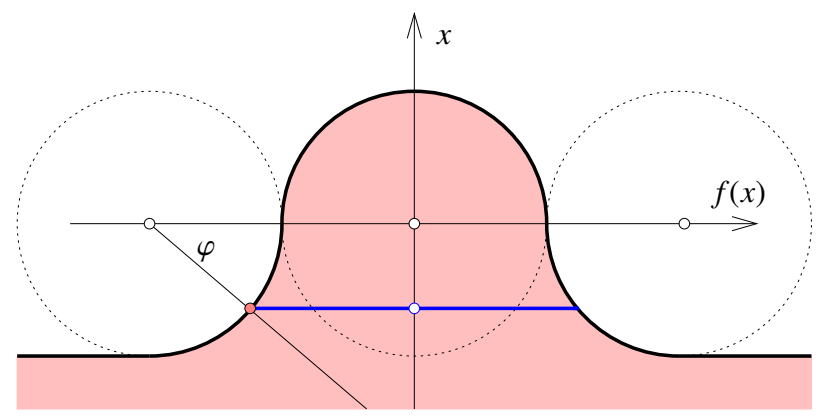

Figure 6: The unit bump obtained by rotating two quarter circles about the vertical axis. The two dotted circles are the cross-sections of the torus delimiting the bump. We compute the two principal curvatures at the solid point by projecting the blue circle to the plane with angle $\varphi$.

Two elementary results. It is easy to see that the total mean curvature over the hemi-sphere is $\operatorname{Mean}_{S}=2 \pi$. The computation of the total mean curvature of the quarter torus requires some preparations. First, we recall the substitution method for integration. Specifically, we set $x=\sin \alpha$, $\mathrm{d} x=\cos \alpha \mathrm{d} \alpha$, and notice that $1 / \sqrt{1-x^{2}}=1 / \cos \alpha$. Hence

$$
\int_{0}^{1} \frac{1}{\sqrt{1-x^{2}}} \mathrm{~d} x=\int_{0}^{\pi / 2} \frac{\cos \alpha}{\cos \alpha} \mathrm{d} \alpha
$$

which gives $\frac{\pi}{2}$. Second, we compute the curvature of an ellipse at the endpoints of its short axis. Assuming the halflength of the long axis is 1 and that of the short axis is $\cos \alpha$, this curvature is $\cos \alpha$. To see this, recall that the unit circle is the best approximating circle of the parabola with formula $y=\frac{1}{2} x^{2}-1$. Imagine the drawing in three dimensions and rotate the plane of the drawing about the horizontal axis until the projection of the circle back to the original plane is the ellipse with desired axes. The projection of the paraboloid satisfies $y=\frac{\cos \alpha}{2} x^{2}-\cos \alpha$. The best approximating circle thus has radius $1 / \cos \alpha$, as required.

Quarter torus. We now compute the area and the total curvature of the quarter torus, obtained by rotating the circle that is the graph of $f(x)=2-\sqrt{1-x^{2}}$. As before, we have $\sqrt{1+f^{\prime}(x)^{2}}=1 / \sqrt{1-x^{2}}$. There area is therefore

$$
\begin{aligned}
\operatorname{Area}_{T} & =2 \pi \int_{-1}^{0} f(x) \sqrt{1+f^{\prime}(x)^{2}} \mathrm{~d} x \\
& =2 \pi \int_{-1}^{0}\left(\frac{2}{\sqrt{1-x^{2}}}-1\right) \mathrm{d} x,
\end{aligned}
$$

which evaluates to $2 \pi(\pi-1)$. To compute the total mean curvature, we first get the two principal curvatures. Along the rotating quarter circle, it is -1 . In the other direction, we get it by projecting the horizontal circle with radius $f(x)$; see Figure 6. The angle of the projection is $\varphi=\arcsin x$. Hence, $x=\sin \varphi$ and $\cos \varphi=\sqrt{1-x^{2}}$. The projection of the circle is an ellipse with axes of half-lengths $f(x)$ and $f(x) \cos \varphi$. It follows that the second principal curvature is $\cos \varphi / f(x)$. We get the total mean curvature by integrating the contributions of the two principal curvatures separately:

$$
\begin{aligned}
\operatorname{Mean}_{T, 1} & =-\frac{1}{2} \operatorname{Area}_{T}, \\
\operatorname{Mean}_{T, 2} & =2 \pi \int_{-1}^{0} f(x) \sqrt{1+f^{\prime}(x)^{2}} \frac{\cos \varphi}{2 f(x)} \mathrm{d} x \\
& =2 \pi \int_{-1}^{0} \frac{1}{2} \mathrm{~d} x
\end{aligned}
$$

which gives $\operatorname{Mean}_{T, 1}=-\pi(\pi-1)$ and $\operatorname{Mean}_{T, 2}=\pi$. We get

$$
\begin{aligned}
\text { Mean }_{\text {bump }} & =\operatorname{Mean}_{S}+\operatorname{Mean}_{T, 1}+\operatorname{Mean}_{T, 2} \\
& =2 \pi-\pi(\pi-1)+\pi
\end{aligned}
$$

which evaluates to $(4-\pi) \pi$, as claimed. 


\section{B Notation}

\begin{tabular}{|c|c|}
\hline $\mathbb{R}^{3}, \mathbb{S}^{2}$ & Euclidean space, 2-sphere \\
\hline$\Gamma, \mathbb{M}=\Gamma_{r}$ & curve, thickened curve \\
\hline$r, \varepsilon, R: \Gamma \rightarrow \mathbb{R}$ & thickening parameters, function \\
\hline$L, \operatorname{vol} \mathbb{M}$ & length of curve, volume of body \\
\hline$r_{0}$ & scale parameter, estimated radius \\
\hline$C_{0}=3.459 \ldots$ & mean curvature contribution of ellipse \\
\hline$P \in \mathcal{P}, u \in \mathcal{U}$ & planes, directions \\
\hline$\varrho, a, \varphi$ & distance, value, angle \\
\hline$\chi(\mathbb{M} \cap P), Q_{i} / c_{i}$ & Euler characteristic, Quermassintegrals \\
\hline$\left(x_{j}\right), N$ & infinite sequence, number of points \\
\hline $\operatorname{Dis}_{\mathcal{B}}(N, X)$ & extreme discrepancy \\
\hline $\operatorname{Dis}_{\mathcal{K}}(N, X)$ & isotropic discrepancy \\
\hline$\#(J, N, X)$ & number of points \\
\hline length $J$ & length \\
\hline$F:[0,1]^{s} \rightarrow \mathbb{R}$ & Euler characteristic function \\
\hline$F^{-1}[c, \infty)$ & superlevel set \\
\hline $\mathbb{M}, m$ & underl. space of simpl. cplx, \#vertices \\
\hline $\operatorname{Err}(N, X)$ & integration error \\
\hline $\operatorname{har}(F, c), \operatorname{Har}(F)$ & \#convex sets, Harman variation \\
\hline$f: \mathbb{M} \rightarrow \mathbb{R}$ & height function \\
\hline $\mathbb{M}_{\alpha}=f^{-1}(-\infty, \alpha]$ & sublevel set \\
\hline $\mathbb{M}^{\alpha}=f^{-1}[\alpha, \infty)$ & superlevel set \\
\hline$c_{i}<\alpha_{i}$ & hom. critical, regular values \\
\hline$H_{i}=\mathrm{H}\left(\mathbb{M}_{\alpha_{i}}\right)$ & absolute homology groups \\
\hline$H_{2 n-i}=\mathrm{H}\left(\mathbb{M}, \mathbb{M}^{\alpha_{i}}\right)$ & relative homology groups \\
\hline$\left(\alpha_{b}, \alpha_{d}\right)$ & birth-death pair \\
\hline $\operatorname{Dgm}_{p}(f)$ & multi-set of birth-death pairs \\
\hline $\mathrm{Up}_{p}(f), \mathrm{Dn}_{p}(f)$ & up, down multi-sets \\
\hline$B_{p}^{k}(f)$ & $k$-th persistence moment \\
\hline$B_{p}^{k}\left(f, r_{0}^{-}\right), B_{p}^{k}\left(f, r_{0}^{+}\right)$ & small, large $k$-th moments \\
\hline$X_{p}^{k}(f), Y_{p_{\sim}^{k}}^{k}(f, g)$ & stabilized moments \\
\hline$Q_{2}, \bar{Q}_{2}, \tilde{Q}_{2}$ & estimates of 2-nd Quermassintegral \\
\hline$C=4+\delta$ & constant exponent \\
\hline$\mu: \mathbb{M} \rightarrow \mathbb{M}^{\prime}$ & homeomorphism \\
\hline$g(x)=f(\mu(x))$ & perturbed height function \\
\hline$\varepsilon=\|f-g\|_{\infty}$ & difference between functions \\
\hline$\varepsilon_{0}=4 \varepsilon / r_{0}$ & normalized difference \\
\hline
\end{tabular}

Table 3: Notation for geometric concepts, sets, functions, vectors, variables used in the paper. 